\title{
Simplified 2-Dimensional Scaled Min-Sum Algorithm for LDPC Decoder
}

\author{
Keol Cho*, Wang-Heon Lee** and Ki-Seok Chung ${ }^{\dagger}$
}

\begin{abstract}
Among various decoding algorithms of low-density parity-check (LDPC) codes, the minsum (MS) algorithm and its modified algorithms are widely adopted because of their computational simplicity compared to the sum-product (SP) algorithm with slight loss of decoding performance. In the MS algorithm, the magnitude of the output message from a check node $(\mathrm{CN})$ processing unit is decided by either the smallest or the next smallest input message which are denoted as $\min 1$ and min2, respectively. It has been shown that multiplying a scaling factor to the output of $\mathrm{CN}$ message will improve the decoding performance. Further, Zhong et al. have shown that multiplying different scaling factors (called a 2-dimensional scaling) to $\min 1$ and $\min 2$ much increases the performance of the LDPC decoder. In this paper, the simplified 2-dimensional scaled (S2DS) MS algorithm is proposed. In the proposed algorithm, we figure out a pair of the most efficient scaling factors which multiplications can be replaced with combinations of addition and shift operations. Furthermore, one scaling operation is approximated by the difference between $\min 1$ and $\min 2$. The simulation results show that S2DS achieves the error correcting performance which is close to or outperforms the SP algorithm regardless of coding rates, and its computational complexity is the lowest comparing to modified versions of MS algorithms.
\end{abstract}

Keywords: Error-correction code, Low-density parity-check code, LDPC decoder, Min-sum algorithm, Normalized min-sum algorithm

\section{Introduction}

Low-density parity-check (LDPC) codes were introduced by Gallager [1], but hardly attracted attention due to its high hardware implementation complexity. However, Mackay and Neal rediscovered advantages of LDPC codes in 1996 [2], and various researches, such as improving error-correcting performance, implementing efficient LDPC decoders, and lowering power consumption of LDPC decoders, have been conducted [3-5]. Due to their near Shannon limit performance [6], easily parallelizable characteristics, and linear decoding complexity, LDPC codes have become popular error correcting codes in many modern communication systems which require faster and higher data rates without any error. LDPC codes have been adopted as forward-error correction (FEC) codes in several emerging communication standards, such as IEEE 802.11n/ac (Wi-Fi) [7, 8], IEEE 802.11ad (WiGig) [9], and IEEE 802.3an (10 Gbase-T Ethernet) [10].

An LDPC code is uniquely defined by an $M$ by $N$ parity check matrix $\mathbf{H}$, where $M$ is the number of the parity checks and $N$ is the length of the codeword. The matrix $\mathbf{H}$ for a binary LDPC code is very sparse with few nonzero elements. As shown in Fig. 1, the $\mathbf{H}$ matrix is also

$\dagger \quad$ Corresponding Author: Dept. of Electronic Engineering, Hanyang University, Seoul, Korea. (kchung@hanyang.ac.kr)

* Dept. of Electronics and Computer Engineering, Hanyang University, Seoul, Korea. (keolman2@gmail.com)

** Dept. of IT, Hansei University, Gunpo-si, Gyeonggi-do, Korea (whlee@hansei.ac.kr)

Received: July 28, 2016; Accepted: December 26, 2016 described by a bipartite graph [11], which is composed of variable nodes (VNs) for columns of the $\mathbf{H}$ matrix in one partite and check nodes (CNs) for rows of the $\mathbf{H}$ matrix in the other. An edge is connected between $\mathrm{VN} i$ and $\mathrm{CN} j$ if the element of the $i$-th column and the $j$-th row is one. The number of VNs connected to a $\mathrm{CN}$ is the degree of $\mathrm{CN}, d_{c}$, and the number of $\mathrm{CNs}$ connected to a $\mathrm{VN}$ is the degree of VN, $d_{v}$. An LDPC code with constant $d_{v}$ and $d_{c}$ is called $\left(d_{v}\right.$, $d_{c}$ )-regular code; otherwise, it is called an irregular code.

The LDPC codes are typically decoded by a messagepassing algorithm, which iteratively exchanges messages through the edges between the CNs and the VNs. In the sum-product (SP) algorithm, also known as the beliefpropagation (BP) algorithm, messages are exchanged in the form of log-likelihood ratios (LLRs) between $\mathrm{CNs}$ and VNs [1]. The SP algorithm achieves a powerful decoding performance close to the Shannon limit, but suffers from high computational complexity. The computational complexity of the SP algorithm can be greatly reduced by using the min-sum (MS) approximation [12], but slight performance loss is incurred. To resolve the performance loss of the MS algorithm, many modified versions of the MS algorithm have been proposed. Most of them have tried to multiply the check to variable node (CTV) messages by a scaling factor to compensate for overestimated belief messages in comparison to the SP algorithm, and thus, these approaches are commonly called normalized MS (NMS) algorithms [13, 14]. In [15], the CTV messages are adjusted by an offset based on the number of VNs connected to the CNs, and the CTV 


$$
H=\left[\begin{array}{llllll}
1 & 0 & 0 & 1 & 0 & 0 \\
0 & 1 & 0 & 0 & 1 & 0 \\
1 & 0 & 1 & 0 & 1 & 0 \\
0 & 0 & 1 & 0 & 0 & 1
\end{array}\right]
$$

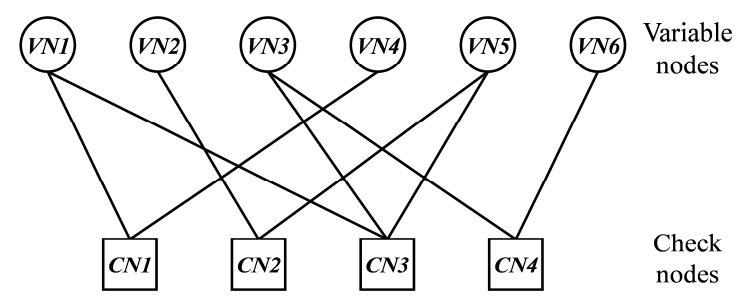

Fig. 1. An example of the H matrix for LDPC code and its bipartite graph representation

messages are adaptively scaled based on the iteration count $[16,17]$. In [18], the first two smallest CTV messages are scaled by different scaling factors using density evolution to improve the decoding performance. Even though the existing algorithms enhance the performance of LDPC decoders, [15, 16], and [18] did not take the hardware implementation cost into account, and [17] suffered from the increased average iteration count until the decoding process is completed.

To estimate the hardware cost of the LDPC decoder, the number of bits to quantize the exchanged messages should be considered because it directly affects both the errorcorrecting capability and the hardware cost of the decoder. The hardware implementation cost typically includes the decoder circuit size and the amount of memory usage. Studies have shown that there is slight performance loss when 5 or 6 bit fixed point representation is used to quantize the message compared to floating point representation [19, 20]. [21] aggressively quantized the CTV message using only 2 bits, but the performance loss was about $0.3 \mathrm{~dB}$. Finding the first two minima among VN to $\mathrm{CN}$ (VTC) messages is usually adopted when MS-based LDPC decoders are implemented in hardware due to the efficiency in memory usage [22]. B. Xiang et al. [23] showed that instead of sending the two found minima, the interconnection complexity and memory usage could be reduced by compressing CTV messages using the difference of the two minima $(\Delta \min )$ which will need a smaller quantization bit width. By this compression, the memory usage is reduced by $5.64 \%$ while the performance loss is up to $0.15 \mathrm{~dB}$.

In this paper, a new decoding algorithm which both the decoding performance and the complexity of hardware implementation of LDPC decoder are taken into account based on the MS algorithm is proposed. As reported in [18], applying two dimensional (2D) scaling factors to the first two minima improves the decoding performance. In the proposed algorithm of this paper, the $2 \mathrm{D}$ scaling factors are determined so that the hardware cost is minimized without losing the decoding performance. In addition, these scaling factors are further optimized by using the $\Delta$ min of the CTV message, and achieves 0.2 to $0.5 \mathrm{~dB}$ coding gain with the least computational complexity compared to modified versions of MS algorithms.

The remainder of this paper is organized as follows. In section 2, representative decoding algorithms of the LDPC code are briefly introduced. The proposed decoding algorithm is explained in detail in section 3 , and the experimental results are described with the complexity analysis in section 4. Finally, our conclusions are presented in section 5 .

\section{Decoding Algorithms of the LDPC Code}

In this section, representative decoding algorithms of the LDPC code will be described. Let us suppose that the LDPC code is defined by an $M$ by $N$ parity check matrix. The set of VNs neighboring $\mathrm{CN} j(j=1,2, \ldots, M)$ is denoted as $V_{j}$, and the set of CNs neighboring $\mathrm{VN} i$ $(i=1,2, \ldots, N)$ is denoted as $C_{i}$. Also, $V_{j} \backslash i$ denotes the subset of VNs excluding the $i$-th $\mathrm{VN}$, and $C_{i} \backslash j$ represents the subset of CNs excluding the $j$-th CN. The LDPC decoder iteratively updates the belief messages and estimates codewords using the following information.

$F_{i} \quad$ : The initial LLR value (a priori LLR) of the $i$-th bit. It is derived from the received vector, $y_{i}$.

$L_{j \rightarrow i}^{(l)}$ : The CTV message; the message sent from $\mathrm{CN} j$ to $\mathrm{VN} i$ at the $l$-th iteration count. It is obtained from the extrinsic VTC messages $L_{i^{\prime} \rightarrow j}$, where $i^{\prime} \in \mathrm{V}_{\mathrm{j}} \backslash i$.

$L_{i \rightarrow j}^{(l)}$ : The VTC message; the message sent from $\mathrm{VN} i$ to $\mathrm{CN} j$ at the $l$-th iteration count. It is obtained from $F_{i}$ and the extrinsic CTV message $L_{j \prime \rightarrow i}$, where $j^{\prime} \in \mathrm{C}_{\mathrm{i}} \backslash$.

$z_{i} \quad$ : The a posteriori LLR of the $i$-th bit computed at each iteration. It is obtained from $F_{i}$ and the information $L_{j \rightarrow i}^{(l)}$

\subsection{Sum-product algorithm}

The SP algorithm assuming that codewords are modulated by binary phase shift keying (BPSK) and they are transmitted over additive Gaussian noise channel (AWGN) with noise variance, $\delta^{2}$ can be described as follows.

- Initialization: For each $i$, a priori LLR and the initial VTC message are updated by

$$
L_{i \rightarrow j}^{(0)}=F_{i}=\frac{2 y_{i}}{\delta^{2}} .
$$

- Iterative steps: For each iteration count $l(l=1, \ldots$, max_iteration) the three following steps are processed.

1) Check node process: For each $j$, $i$, update CTV message by 


$$
L_{j \rightarrow i}^{(l)}=2 \tanh ^{-1}\left(\prod_{i^{\prime} \in V_{j} \backslash i} \tanh \left(\frac{L_{i^{\prime} \rightarrow j}^{(l-1)}}{2}\right)\right) .
$$

2) Variable node process: For each $j$, $i$, update VTC message by

$$
L_{i \rightarrow j}^{(l)}=F_{i}+\sum_{j^{\prime} \in C_{i} \backslash j} L_{j^{\prime} \rightarrow i}^{(l)},
$$

and update a posteriori LLR by

$$
z_{i}=F_{i}+\sum_{j \in C(i)} L_{j \rightarrow i}^{(l)}
$$

3) Tentative decision and stopping criterion test:

i) In the tentative decision, the estimated codeword $\hat{\boldsymbol{c}}=\left\{\hat{c}_{1}, \hat{c}_{2}, \ldots, \hat{c}_{N}\right\}$ is constructed based on $z_{i}$ by

$$
\hat{\boldsymbol{c}}=\left\{\hat{c}_{1}, \hat{c}_{2}, \ldots, \hat{c}_{N}\right\}, \text { where } \hat{c}_{i}= \begin{cases}0, & z_{i} \geq 0 \\ 1, & z_{i}<0\end{cases}
$$

ii) If either the syndrome check, $\mathbf{H} \cdot \hat{\boldsymbol{c}}^{T}=0$, or the number of iterations reaches the predefined maximum count, $\hat{\boldsymbol{c}}$ becomes the output of the decoder. When the syndrome check is not satisfied, the decoder goes back to 2) and increments the iteration count.

\subsection{Min-sum algorithms}

In the MS algorithm, (3), (4), and (5) are equivalent to the SP algorithm. Instead of (1), the MS algorithm initializes $F_{i}$ and $L_{j \rightarrow i}^{(0)}$ with

$$
L_{i \rightarrow j}^{(0)}=F_{i}=y_{i}
$$

and the $\mathrm{CN}$ process (3) is approximated by the minimum finding function as follows:

$$
L_{j \rightarrow i}^{(l)}=\prod_{i \in V_{j} \backslash i} \operatorname{sign}\left(L_{i^{\prime} \rightarrow j}^{(l-1)}\right) \min _{i^{\prime} \in V_{j} \backslash i}\left|L_{i^{\prime} \rightarrow j}^{(l-1)}\right|
$$

The approximation of the CTV message in the MS algorithm is known to be overestimated compared to the CTV message of the SP algorithm, [13] and [14] normalize the CTV message using scaling factor, $\alpha$. With the normalization, (7) is rewritten by

$$
L_{j \rightarrow i}^{(l)}=\alpha \prod_{i \in V_{j} \backslash i} \operatorname{sign}\left(L_{i \rightarrow j}^{(l-1)}\right) \min _{i \in V_{j} \backslash i}\left|L_{i \rightarrow j}^{(l-1)}\right|
$$

It is proven that the optimal $\alpha$ varies according to the code rate, the signal-to-noise (SNR) ratio, and the codeword length [24]. Therefore, various researches have followed in order to figure out $\alpha$ for the best error correcting performance or for the most efficient hardware implementation [15-18, 20].

In [15], degree match two-step MS (DM2S) has been proposed. DM2S compensates CTV messages by subtracting positive correction factors, which is derived by logarithmic calculation of $d_{c}$. In DM2S, CNs have different correction factors according to their own $d_{c}$ 's, and the steps are decided by the magnitude of the smallest LLR and the distance between the first two smallest magnitudes of LLRs.

[16] and [17] adaptively adjust $\alpha$ according to the iteration count of the decoding process, and the adjustment is based on the fact that the reliability of the LLRs is improved as the iteration count goes up. Generalized simplified variable-scaled MS (GSVS) algorithm [17] divides the iteration count into four steps and the initial scaling factor $\alpha_{0}$ is increased as follows: $\alpha_{0}, 0.5+0.5 \cdot \alpha_{0}$, $0.75+0.25 \cdot \alpha_{0}$, and $0.875+0.125 \cdot \alpha_{0}$.

In (7), the minimum should be found for all of the neighboring VNs excluding the $i$-th $\mathrm{VN}$, which implies that the magnitude of CTV message is either the smallest LLR or the next smallest LLR. Thus, the minimum finding part in (7) can be replaced by

$$
\min _{i^{\prime} \in V_{j} \backslash i}\left|L_{i^{\prime} \rightarrow j}^{(l)}\right|=\left\{\begin{array}{lr}
\min 2, & \text { if the index of } \min 1=i \\
\min 1, & \text { otherwise }
\end{array}\right.
$$

where $\min 1$ and $\min 2$ are the first and the second minimum, respectively. Zhong et al. [18] have shown that 2-dimensional scaling (2DS) of $\min 1$ and $\min 2$ by $\alpha_{1}$ and $\alpha_{2}\left(0<\alpha_{1}<\alpha_{2}<1.0\right)$, respectively, achieves almost $0.4 \mathrm{~dB}$ coding gain compared to a single scaling factor. However, the scaling factors of 2DS and DM2S do not consider the hardware implementation cost of the LDPC decoder.

In order to reduce the implementation complexity, a modified 2DS MS algorithm, called simplified 2dimensional scaled (S2DS) MS algorithm, is proposed in this paper. First of all, scaling factors for 2-dimensional MS which is called hardware considered 2D scaling factors (H-2DS) are chosen to achieve less complex decoder implementation. Further, the computational complexity of the scaling operation is reduced in S2DS using the $\Delta$ min approximation, which has been proposed in [23].

\section{Simplified 2-Dimensional Scaled Min-sum Algorithm}

\subsection{Low complexity $2 D$ scaling factors}

The optimal scaling factors of 2DS vary according to the SNR [18]. Without considering the cost of the hardware implementation of LDPC decoder, 2DS requires multiplying $\min 1$ and $\min 2$ by $\alpha_{1}=0.4902$ and $\alpha_{2}=0.9174$, respectively. It is obvious that multiplying the two minima 
by these scaling factors is quite complicated in aspect of hardware implementation. To figure out the best H-2DS which will require less complex computational complexity, we have carried out simulations with varying $\alpha_{1}$ and $\alpha_{2}$ from 0.5 to 1.0 with increments of 0.125 . It should be noted that multiplication the two minima by these scaling factors can be implemented with the combination of add and shift operations. For the simulation, the subsets of LDPC codes defined in IEEE 802.11ad applications [9], which are irregular and have block length $N$ and dimension $K,(N, K)=(672,336)$ and $(672,504)$, is chosen. For a regular LDPC code, $(408,204)$ code with $\left(d_{v}, d_{c}\right)=(3,6)$ is chosen. The maximum iteration count is set to 20 , and $a$ priori LLRs and the exchanging LLRs have been simulated using the floating point arithmetic. The simulation with the BPSK transmission of the all-zero codeword over the AWGN channel was carried out until the number of frame errors reached at least 100 .

Through the simulations, it turns out that the best performance is achieved with $\alpha_{1}=0.75$ and $\alpha_{2}=0.875$, and

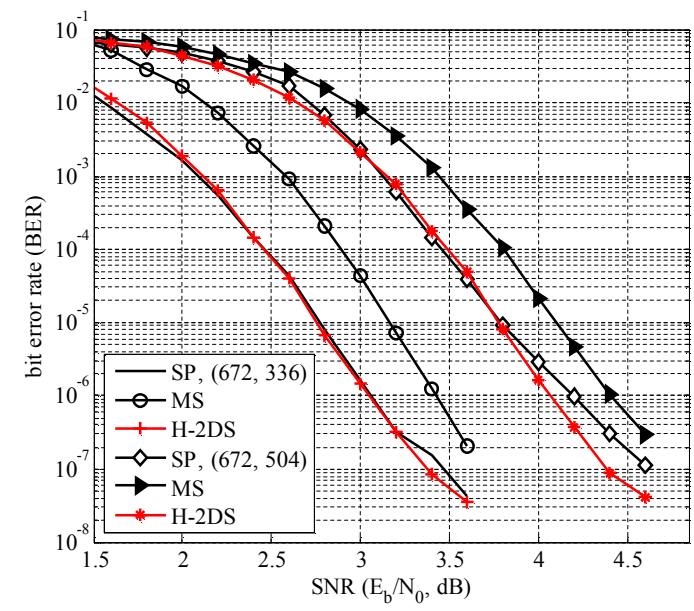

(a) $(672,336)$ and $(672,504)$ irregular codes

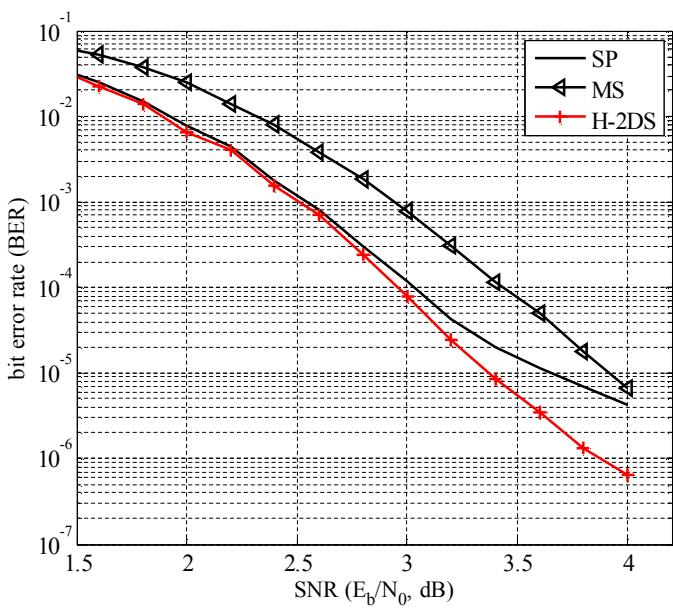

(b) $(408,204)$ regular code $\left(d_{v}, d_{c}\right)=(3,6)$

Fig. 2. BER performance of hardware-considered 2DS the corresponding results of the bit-error rate (BER) performance comparison with the SP and MS algorithms are depicted in Fig. 2. The solid lines and the dashed lines in Fig. 2(a) represent the BER simulation results of the $(672,336)$ code and the $(672,504)$ code, respectively, and Fig. 2(b) shows the result of the $(408,204)$ regular LDPC code. Regardless of the degree regularity and the code rate of the LDPC code, H-2DS achieves coding gains from 0.4 $\mathrm{dB}$ to $0.6 \mathrm{~dB}$ compared to the MS algorithm at BER of $10^{-5}$, and H-2DS even outperforms the SP algorithm in high SNR regions. As aforementioned, multiplications with 0.75 and 0.875 can be implemented by a combination of add and shift operations. The scaling $x$ with 0.75 can be implemented as $0.75 \cdot x=x / 2+x / 4$ and the scaling $x$ with 0.875 can be implemented as $0.875 \cdot x=x / 2+x / 4+x / 8$. According to (8) and (9), the magnitude of the CTV message of the normalized MS algorithm is calculated as follows

$$
\left|L_{j \rightarrow i}^{(l)}\right|=\left\{\begin{array}{lr}
\alpha_{2} \cdot \min 2, & \text { if the index of } \min 1=i \\
\alpha_{1} \cdot \min 1, & \text { otherwise }
\end{array}\right.
$$

and the CTV message consists of \{signs, index of min 1 , $\left.\alpha_{1} \cdot \min 1, \alpha_{2} \cdot \min 2\right\}$. When a single scaling factor is used, $\alpha_{1}$ of (10) is equal to $\alpha_{2}\left(\alpha_{1}=\alpha_{2}=\alpha\right)$, which means that it is still required two multiplications (or few shifts and addition) for scaling in the $\mathrm{CN}$ unit ( $\min 1$ and $\min 2$ with $\alpha$ ). On the other hand, H-2DS requires only shift and add operations, and due to the fact that the scaling factor 0.875 can be computed with only one more add and shift operations in addition to computing the scaling factor 0.75 , only one arithmetic circuit can be used for both scaling operations in a serial manner to save the hardware implementation cost.

\subsection{Simplified 2D scaling with $\Delta \mathrm{min}$}

Based on the pair of scaling factors of H-2DS, the proposed S2DS decoding algorithm further reduces the computational complexity using $\Delta$ min information [23]. In [23], the CTV message is compressed using the difference between $\min 1$ and $\min 2, \Delta \min (=\min 2-\min 1)$. Thus, a CTV message consists of ssigns, index of $\min 1, \min 1$, $\Delta \min \}$, which reduces the memory usage and the interconnection complexity of the LDPC decoder compared to sending $\min 1$ and $\min 2$.

Utilizing $\Delta \min$ information, S2DS replaces the scaling operation, $0.875 \cdot \min 2$ by the following computation under the assumption that the magnitudes of $\min 1$ and $\Delta \min$ are similar:

$$
\begin{aligned}
0.875 \cdot \min 2 & =0.875 \cdot(\min 1+\Delta \min ) \\
& =0.875 \cdot \min 1+0.875 \cdot \Delta \min \\
& =0.75 \cdot \min 1+0.125 \cdot \min 1+0.875 \cdot \Delta \min \\
& \approx 0.75 \cdot \min 1+\Delta \min (i f f . \min 1 \approx \Delta \min )
\end{aligned}
$$




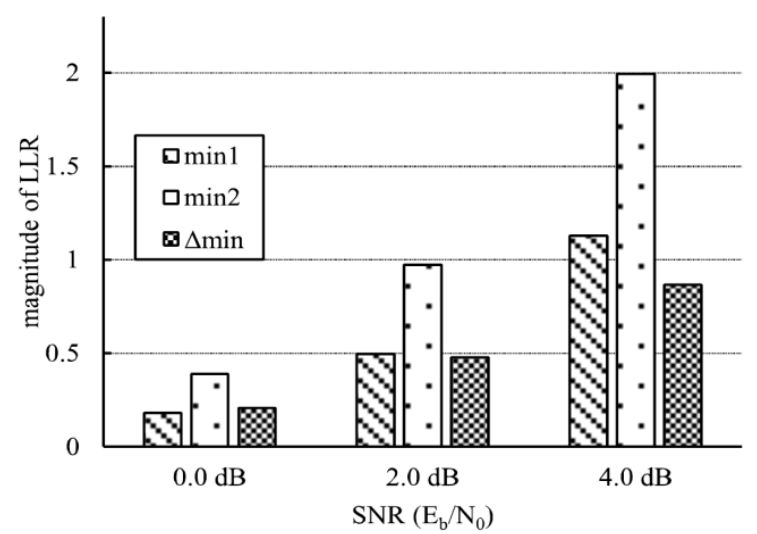

Fig. 3. The average magnitudes of $\min 1, \min 2$, and $\Delta \min$

Under this approximation, the CTV message of S2DS consists of $\{$ signs, index of $\min 1,0.75 \cdot \min 1, \Delta \min \}$, and S2DS requires only one scaling operation in the $\mathrm{CN}$ unit instead of two.

To prove that the above assumption is valid, we have carried out an analysis that compares the magnitudes of $\min 1, \min 2$, and $\Delta \min$. The $(672,546)$ code in IEEE 802.11ad [9] over AWGN channel was chosen for the analysis, and the average magnitudes of $\min 1, \min 2$, and $\Delta$ min of 500,000 codewords were calculated varying SNRs. The analysis results are summarized in Fig. 3, which shows that the magnitude of $\min 1$ is similar to that of $\Delta \mathrm{min}$.

\subsection{Fixed-point implementation}

To reduce the complexity of a hardware implementation, many digital circuits are designed to handle only the fixed point numbers. However, such fixed point implementations should be concerned about the amount of the quantization error that will result in a performance degradation. In this paper, the BER performance of the S2DS algorithm is estimated with a fixed-point Qm. $f$, where an $(m+f)$-bit fixed-point LLR message consists of $m$ integer bits and $f$ fractional bits. The exchanged LLR messages are quantized with 5 bits excluding the sign bit because 5 bit quantization gives the best tradeoff between the performance and the hardware cost [24]. By varying $m$ and $f$, we have carried out simulations to figure out which Qm.f within 5 bits (Q1.4, Q2.3, Q3.2, and Q4.1) shows the best performance.

The simulations have been conducted under the same conditions in section 3.1 . The $(672,336)$ code and the $(672$, 504) code are modulated in BPSK and are transmitted over the AWGN channel with 20 as the maximum iteration count. The BER performance comparison results of the S2DS algorithm with 5 bit quantization are shown in Fig. 4, where the solid lines are for the $(672,336)$ code and the dashed lines are for the $(672,504)$ code. For both codes, Q1.4 and Q4.1 suffer from significant performance degradation, so the results are not depicted in Fig. 4, whereas Q2.3 shows the best decoding performance and does not suffer from an error floor at high SNR regions

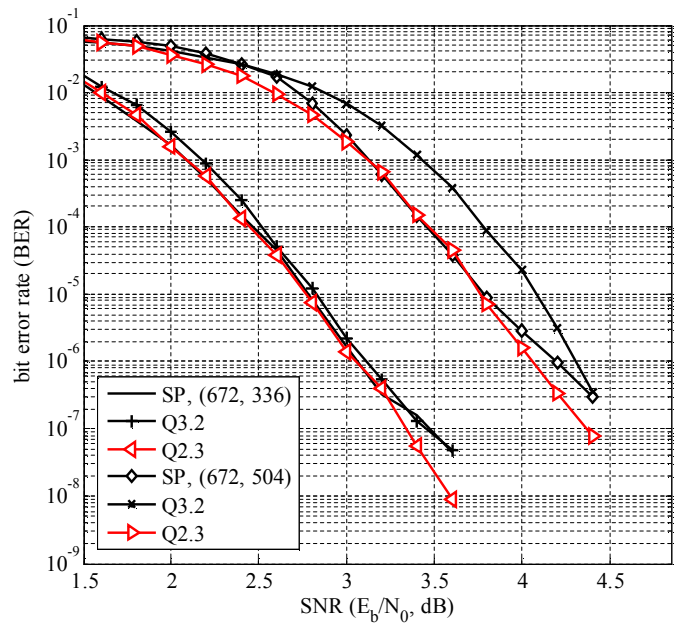

Fig. 4. BER performance of S2DS algorithm in Q3.2 and Q2.3 with SP algorithm in floating point arithmetic

compared to SP and Q3.2.

\section{Simulation Results}

The simulation results are presented to show the performance of the S2DS algorithm by comparing with the simulation results are presented to show the performance of the S2DS algorithm by comparing with the other MSbased algorithms that were briefly mentioned in the paper: SP, MS, GSVS, and DM2S algorithms. The complexity of the proposed algorithm is also discussed in this section.

All of four $\mathbf{H}$ matrices defined in IEEE 802.11ad applications [9] are used for the simulation. These four LDPC codes are irregular and consist of rates $1 / 2,3 / 4,5 / 8$, and 13/16 with a common length of 672 bits: $(672,336)$, $(672,504),(672,420)$, and $(672,546)$ codes, respectively. BPSK transmission of the all-zero codeword over the AWGN channel was used. Simulations were running until at least 400 frame errors were counted at low and middle SNR simulation points and 100 frame errors for high SNR points. The maximum allowable iteration count was set to 20 .

The SP and DM2S algorithms were simulated using a floating-point arithmetic, and the MS and GSVS were simulated using a Q4.6 fixed-point arithmetic. The initial scaling factor, $\alpha_{0}$, of GSVS was set to 0.5 . For each iteration step, the following scaling factors were used: 0.5 , $0.75,0.875$, and 0.9375 .

\subsection{Decoding performance}

The simulation results are compared with the S2DS with Q2.3. As shown in Fig. 5, S2DS algorithm shows the best performance among all the MS-based decoding algorithms. Fig. 5(a), (c), and (d) shows that S2DS achieves the performance close to the SP performance and does not 
Table 1. Computational complexity of a check node within a single iteration

\begin{tabular}{|c|c|c|c|c|c|c|c|}
\hline Decoding algorithms & Multiplication & Division & Comparison & Addition & Subtraction & Bit shift & Remarks \\
\hline SP & $d_{c}$ & $d_{c}-1$ & 0 & 0 & 0 & 0 & $\tanh , \tanh ^{-1}$ \\
\hline MS & 0 & 0 & $d_{c}+\log _{2} d_{c}-2$ & 0 & 0 & 0 & - \\
\hline NMS (0.75) & 0 & 0 & $d_{c}+\log _{2} d_{c}-2$ & 2 & 0 & 4 & - \\
\hline DM2S & 0 & 0 & $d_{c}+\log _{2} d_{c}+2$ & 2 & 3 & 4 & logarithm \\
\hline GSVS & 0 & 0 & $d_{c}+\log _{2} d_{c}-1$ & 7 & 0 & 9 & iteration counter \\
\hline S2DS & 0 & 0 & $d_{c}+\log _{2} d_{c}-2$ & 1 & 1 & 2 & - \\
\hline
\end{tabular}

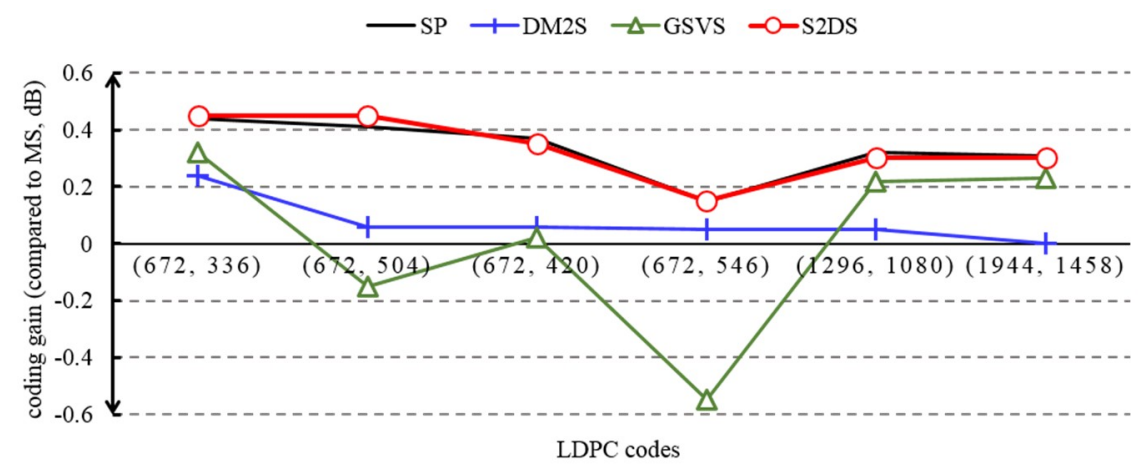

Fig. 6. Coding gain compared to MS at a BER of $10^{-5}$ with various LDPC codes: $\mathrm{N}=672$ codes in [9], $\mathrm{N}=1296$ and $\mathrm{N}=1944$ in [7]

suffer from an error floor at high SNR points. In the case of high coding rates as shown in Fig. 5 (b), S2DS outperforms SP. It is proven that the SP algorithm does not provide the optimal decoding method in short code lengths [13].

For more precise comparison of the decoding performance, the coding gains compared to MS at a BER of $10^{-5}$ have been analyzed with two more LDPC codes in [7] which have different code lengths $(\mathrm{N}=1296$ and $\mathrm{N}=1944)$ and dimensions with those of in Fig.5. As shown in Fig. 6 which depicts the coding gains for various LDPC codes, S2DS achieves overall coding gain close to SP (less than $0.02 \mathrm{~dB}$ ) and more than DM2S (from $0.07 \mathrm{~dB}$ to $0.3 \mathrm{~dB}$ ). However, the coding gains of GSVS fluctuate with codes. It implies that it is crucial to figure out the best performing scaling factor sets for GSVS, which requires more computational logics for sets of scaling factors. In contrast, S2DS uses a constant scaling factor and achieves much better performance.

More aggressive quantization of $\Delta \mathrm{min}$ information was also examined. However, Q1.2 and Q2.1 for $\Delta$ min showed significant performance degradation. Q2.2 for $\Delta \mathrm{min}$ showed a performance drop about $0.05 \mathrm{~dB}$, but suffered from early error floors at high SNR points.

\subsection{Computational complexity}

In this section, the computational complexity of various decoding algorithms is analyzed. Table 1 summarizes the computational complexity of the $\mathrm{CN}$ processing in each iteration. The set of the basic arithmetic operations is listed with extra operations besides the basic operations in the remarks. For example, the SP algorithm requires floating point multiplications and divisions including the hyperbolic tangent calculation. The comparison column shows the required number of comparisons to find the first two minimum values $\left(d_{c}+\log _{2} d_{c}-2\right)$ for $\mathrm{MS}$ algorithm [22].

The MS algorithm requires the least amount of computation among all of the decoding algorithms, and S2DS requires the second least. DM2S requires more computations including natural logarithms for the thresholds and their subjects. GSVS requires quite a few combinations of shift and addition operations to compute the scaling factor sets, and an additional counter and a comparator are required for choosing the step that decides which scaling factors will be used. However, S2DS requires only a single scaling factor that is computed by two shift operations and one addition, and a $\Delta \mathrm{min}$ information obtained from one subtraction. Furthermore, since the scaling operation of S2DS remains the same for different coding rates, only one scaling unit is required when a multi-rate LDPC decoder is implemented. Considering that SP requires multiplications and divisions and DM2S requires divisions and logarithmic computations, S2DS can be claimed to the best when both the computational complexity and the decoding performance are taken into account.

\section{Conclusion}

This paper proposed a simple yet powerful 2dimensional scaled min-sum algorithm called S2DS minsum algorithm. We figure out scaling factors with which 


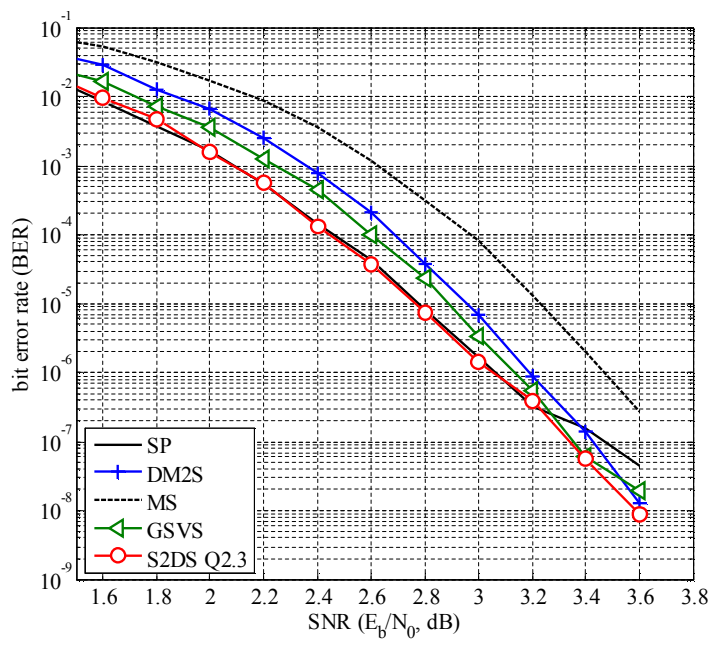

(a) $(672,336)$ code

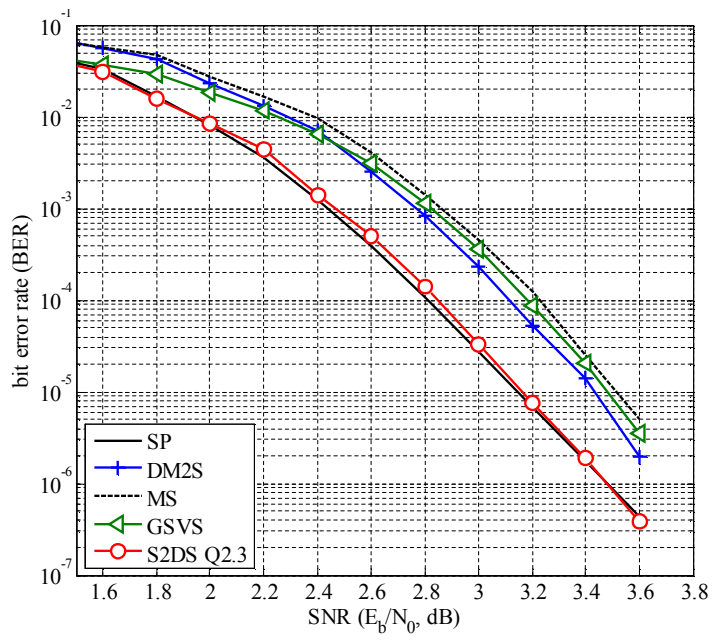

(c) $(672,420)$ code

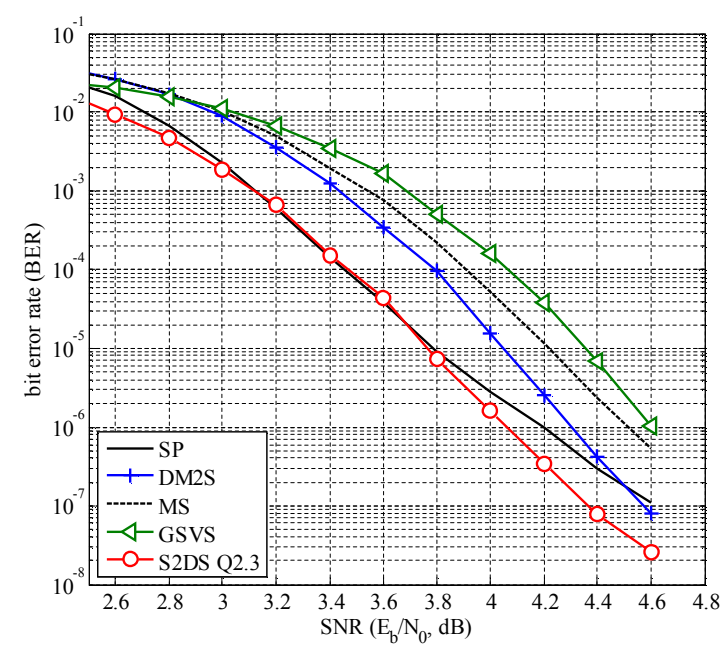

(b) $(672,504)$ code

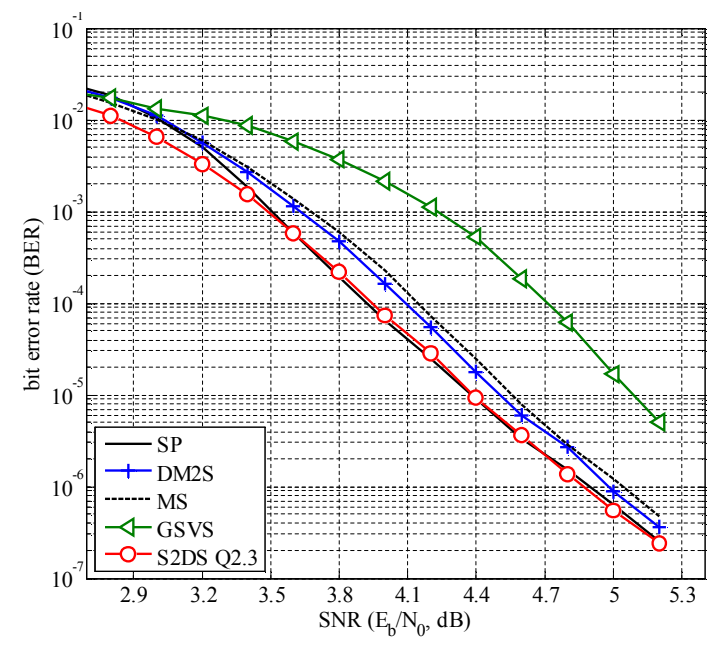

(d) $(672,546)$ code

Fig. 5. Decoding performance of Q2.3 S2DS compared with SP, DM2S, MS, and GSVS

scaling operation can be simplified. Further, we show that one scaling operation is approximated by the difference between $\min 1$ and $\min 2$. Therefore, our proposed algorithm reduced the complexity of the check node computation significantly. In spite of the simplicity, the proposed S2DS algorithm achieves coding gains from $0.2 \mathrm{~dB}$ to $0.4 \mathrm{~dB}$ compared to the other min-sum based decoding algorithms, and its performance is consistently good regardless of the coding rate or the irregularity of the LDPC codes.

\section{Acknowledgements}

This research was supported by Basic Science Research Program through the National Research Foundation of Korea(NRF) funded by the Ministry of Education(NRF2015R1D1A1A09061079).

\section{References}

[1] Robert G. Gallager, "Low-Density Parity-Check Codes," IRE Trans. Information Theory, vol. 8, no. 1, pp. 21-28, Jan. 1962.

[2] D. J. C. MacKay and R. M. Neal, "Near Shannon Limit Performance of Low Density Parity Check Codes," IET Electron. Lett., vol. 32, no. 18, pp. 1645, Aug. 1996.

[3] L. Li, D. Qu, and T. Jiang, "Partition Optimization in LDPC-Coded OFDM Systems with PTS PAPR Reduction," IEEE Trans. Veh. Technol., vol. 63, no. 8, pp. 4108-4113, Oct. 2014.

[4] A. J. Wong, S. Hemati, and W. J. Gross, "Efficient Implementation of Structured Long Block-Length LDPC Codes," in 2015 IEEE 26th International Conference on Application-specific Systems, Architectures and Processors (ASAP), Toronto, Canada, July 2015. 
[5] Y. S. Park, D. Blaauw, D. Sylvester, and Z. Zhang, "Low-Power High-Throughput LDPC Decoder Using Non-Refresh Embedded DRAM," IEEE J. Solid-State Circuits, vol. 49, no. 3, pp. 783-794, Mar. 2014.

[6] Sae-Young Chung, G. D. Forney, T. J. Richardson, and R. Urbanke, "On the Design of Low-Density Parity-Check Codes within $0.0045 \mathrm{~dB}$ of the Shannon Limit," IEEE Commun. Lett., vol. 5, no. 2, pp. 58-60, Feb. 2001.

[7] Wireless LAN medium access control (MAC) and physical layer (PHY) specifications: enhancements for higher throughput, IEEE Std. P802.11n/D7.0, 2008.

[8] IEEE 802.11ac-Enhancements for Very High Throughput for operation in bands below $6 \mathrm{GHz}$, IEEE P802.11ac/D5.0, 2013.

[9] IEEE P802.11ad: Wireless LAN Medium Access Control (MAC) and Physical Layer (PHY) specifications - Enhancements for Higher Throughput in the $60 \mathrm{GHz}$ Band, IEEE, 2012.

[10] Carrier Sense Multiple Access with Collision Detection (CSMA/CD) Access Method and Physical Layer Specifications, IEEE Std. 802.3an, 2006.

[11] R. Tanner, "A Recursive Approach to Low Complexity Codes," IEEE Trans. Inform. Theory, vol. 27, no. 5, pp. 533-547, Sep. 1981.

[12] J. Hagenauer, E. Offer, and L. Papke, "Iterative Decoding of Binary Block and Convolutional Codes,' IEEE Trans. Inf. Theory, vol. 42, no. 2, pp. 429-445, Mar. 1996.

[13] J. Chen and M. Fossorier, "Near Optimum Universal Belief Propagation Based Decoding of Low Density Parity Check Codes," IEEE Trans. Commun., vol. COM-50, no. 3, pp. 406-414, Mar. 2002.

[14] J. Heo, "Analysis of Scaling Soft Information on Low Density Parity Check Code," IET Electron. Lett., vol. 39, no. 2, pp. 219, Jan. 2003.

[15] S. L. Howard, C. Schlegel, and V. C. Gaudet, "Degree-Matched Check Node Decoding for Regular and Irregular LDPCs," IEEE Trans. Circuits Syst. II Express Briefs, vol. 53, no. 10, pp. 1054-1058, Oct. 2006.

[16] Y. Xu, L. Szczecinski, B. Rong, F. Labeau, D. He, Y. $\mathrm{Wu}$, and $\mathrm{W}$. Zhang, "Variable LLR Scaling in MinSum Decoding for Irregular LDPC Codes," IEEE Trans. Broadcast., vol. 60, no. 4, pp. 606-613, Dec. 2014.

[17] Ahmed A. Emran and Maha Elsabrouty "Generalized Simplified Variable-Scaled Min Sum LDPC Decoder for Irregular LDPC Codes," Personal, Indoor, and Mobile Radio Communication (PIMRC), 2014 IEEE 25th Annual International Symposium on, Washington DC, USA, Sep. 2014.

[18] Zhou Zhong, Shuming Guo, Xiangyang Xu, and Huiqing Bai, "A Classified Normalized BP-Based
Algorithm with 2-Dimensional Correction for LDPC Codes," Journal of Communications, vol. 8, no. 5, May 2013

[19] R. Zarubica, R. Hinton, S. G. Wilson, and E. K. Hall, "Efficient Quantization Schemes for LDPC Decoders," in IEEE Military Communications Conference, San Diego, Nov. 2008.

[20] J. Zhao, F. Zarkeshvari, and A. H. Banihashemi, "On Implementation of Min-Sum Algorithm and Its Modifications for Decoding Low-Density ParityCheck (LDPC) Codes," IEEE Trans. Commun., vol. 53, no. 4, pp. 549-554, Apr. 2005.

[21] V. A. Chandrasetty and S. M. Aziz, "FPGA Implementation of High Performance LDPC Decoder Using Modified 2-Bit Min-Sum Algorithm," in 2010 Second International Conference on Computer Research and Development, Kuala Lumpur, May 2010.

[22] C. L. Wey, M. D. Shieh and S. Y. Lin, "Algorithms of Finding the First Two Minimum Values and Their Hardware Implementation," IEEE Trans. Circuits Syst. I, vol. 55, no. 11, pp. 3430-3437, Dec. 2008.

[23] B. Xiang, R. Shen, A. Pan, D. Bao and X. Zeng, "An Area-Efficient and Low-Power Multirate Decoder for Quasi-Cyclic Low-Density Parity-Check Codes," IEEE Trans. Very Large Scale Integration Systems, vol. 18, no. 10, pp. 1447-1460, Sep. 2010.

[24] J. Chen, A. Dholakia, E. Eleftheriou, M. P. C. Fossorier, and Xiao-Yu Hu, "Reduced-Complexity Decoding of LDPC Codes," IEEE Trans. Commun., vol. 53, no. 8, pp. 1288-1299, Aug. 2005.

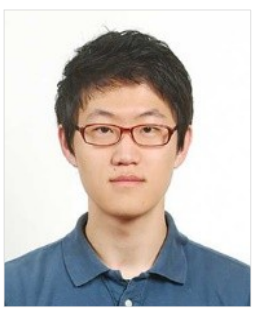

Keol Cho He received his B.S. degree in Media Communication Engineering from Hanyang University, Seoul, Korea in 2009 and his Ph.D. degree in Electronics and Computer Engineering from Hanyang University, Seoul, Korea in 2017. His research interests include system-on-chip architecture, error-correction codes, and hardware implementation of error-correction codes.

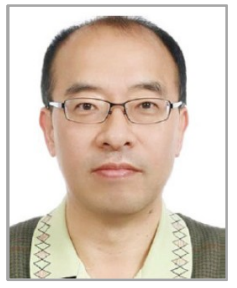

Wang-Heon Lee He received his B.S. degree in Control and Instrumentation from Seoul National University in 1985, his M.S. and Ph.D. degrees in Automation and Design Engineering from Korea Advanced Institute of Science and Technology in 1992, 2001,respectively. In 2006, he joined the Department of Information Technology at the Hansei University, now an associate professor and became a chairman of technical committee of 
Machine Vision from 2011 of ICROS. His research interests include Deep Learning, Mobile Robot Control and Control system design of Drone.

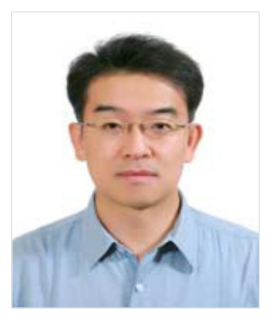

Ki-Seok Chung He received his B.E. degree in Computer Engineering from Seoul National University, Seoul, Korea, in 1989 and his Ph.D. in Computer Science from the University of Illinois at Urbana-Champaign in 1998. Currently, he is a Professor at Hanyang University, Seoul, Korea. His research interests include low-power embedded system design, multi-core architecture, image processing, reconfigurable processor and DSP design, SoC-platform-based verification, and system software for MPSoC. 